Supporting information

\title{
Multimodal nanoscopic study of atomic diffusion and related localized optoelectronic response of $\mathrm{WS}_{2} / \mathrm{MoS}_{2}$ lateral heterojunctions
}

Jiaqi Shao ${ }^{1 \ddagger}$, Fei Chen ${ }^{1 *}$, Weitao Su ${ }^{1,2 *}, \quad$ Yijie Zeng ${ }^{2}$, Hong-Wei Lu ${ }^{2}$

${ }^{1}$ College of Materials and Environmental Engineering, Hangzhou Dianzi University, 310018, Hangzhou, China

${ }^{2}$ School of Sciences, Hangzhou Dianzi University, 310018, Hangzhou, China

\$These authors contributed equally to the paper.

* Corresponding author: suweitao@hdu.edu.cn 


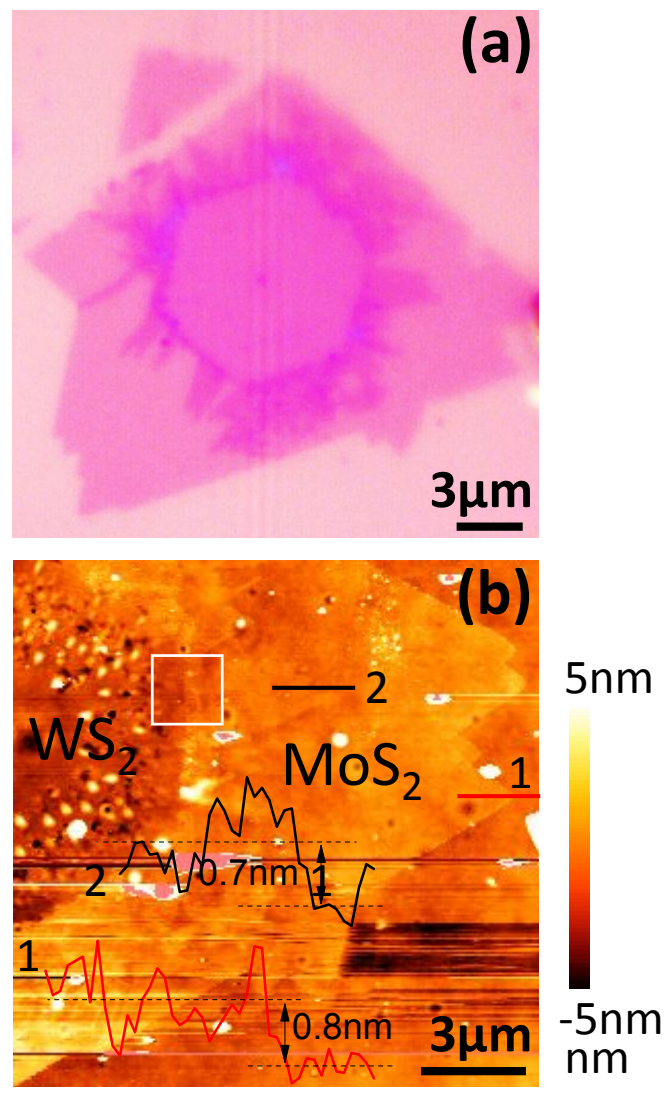

Figure S1 (a)Optical image and (b) AFM topography image of multilayer $\mathrm{WS}_{2} / \mathrm{MoS}_{2}$ HJ II ; The height profiles along line 1 and 2 are plot as insets. 

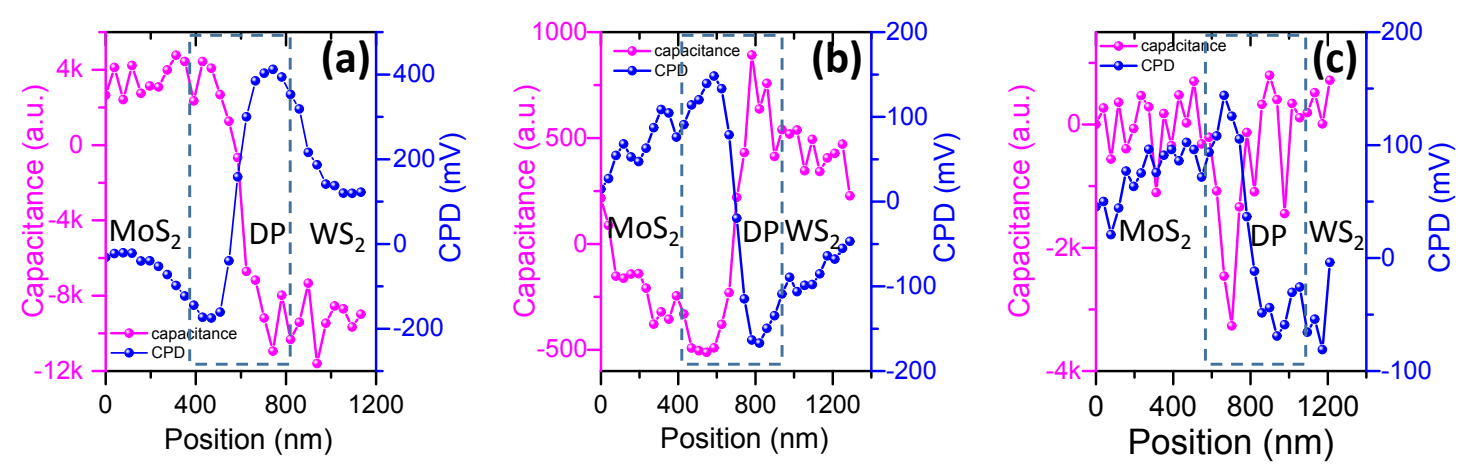

Figure S2 CPD and Capacitance collected along the mark lines in Figure 3: (a) without light; with lasers (b) $632.8 \mathrm{~nm}$ and (c) $532 \mathrm{~nm}$. 


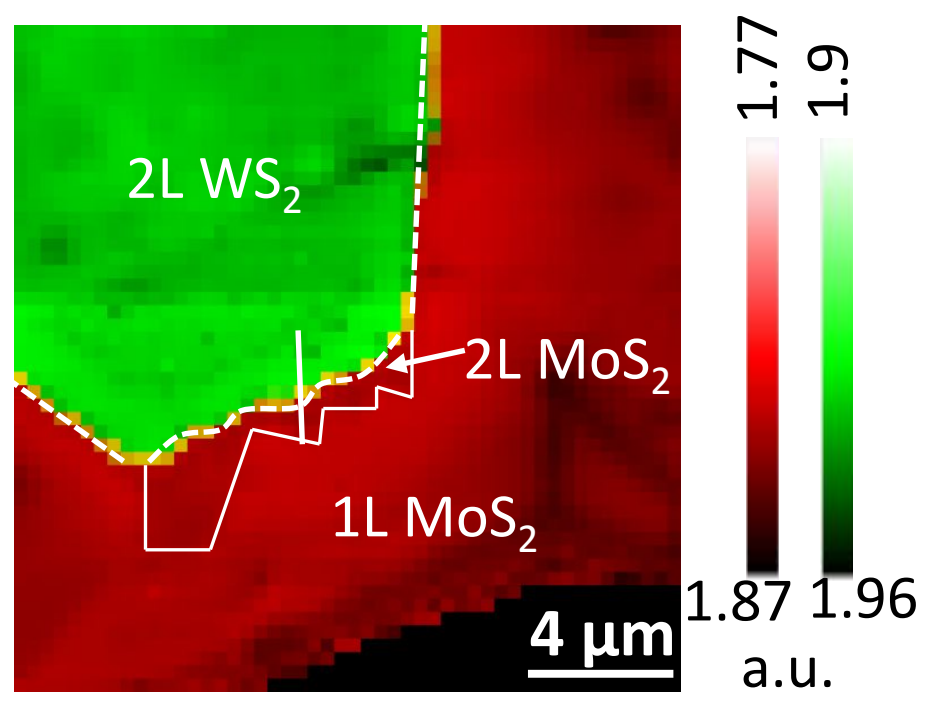

Figure S3 Overlay far field PL energies of $A$ excitons of $\mathrm{WS}_{2}$ (green) and $\mathrm{MoS}_{2}$ (red) of HJ I . The excitation laser wavelength is $532 \mathrm{~nm}$. 

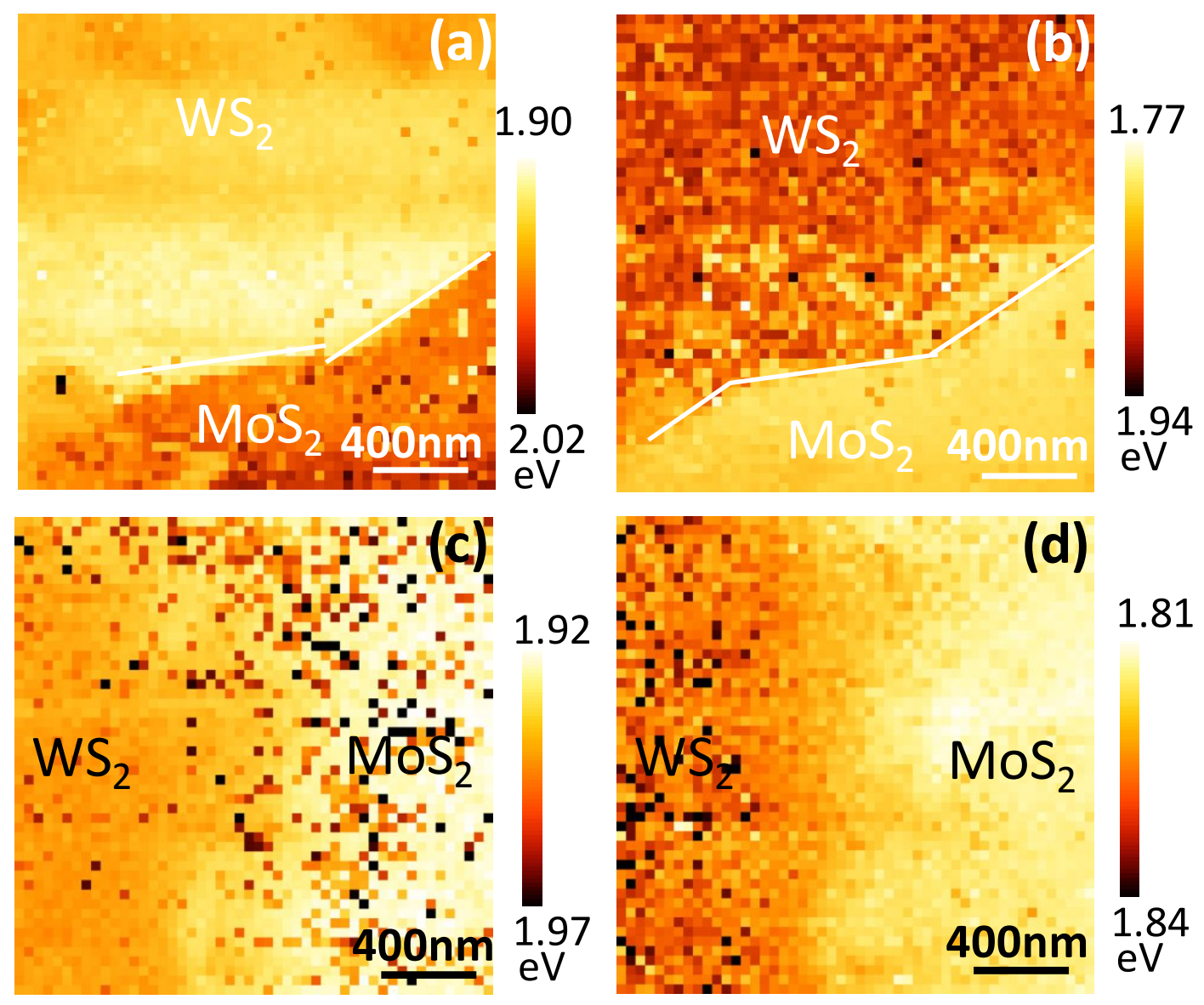

Figure S4 TEPL $A$ excitonic energy images of $\mathrm{WS}_{2}(\mathrm{a})$ and $\mathrm{MoS}_{2}(\mathrm{~b})$ corresponding to Figure 5(f); TEPL $A$ excitonic energy images of $\mathrm{WS}_{2}$ (c) and $\mathrm{MoS}_{2}$ (d) corresponding to Figure 5(h). 


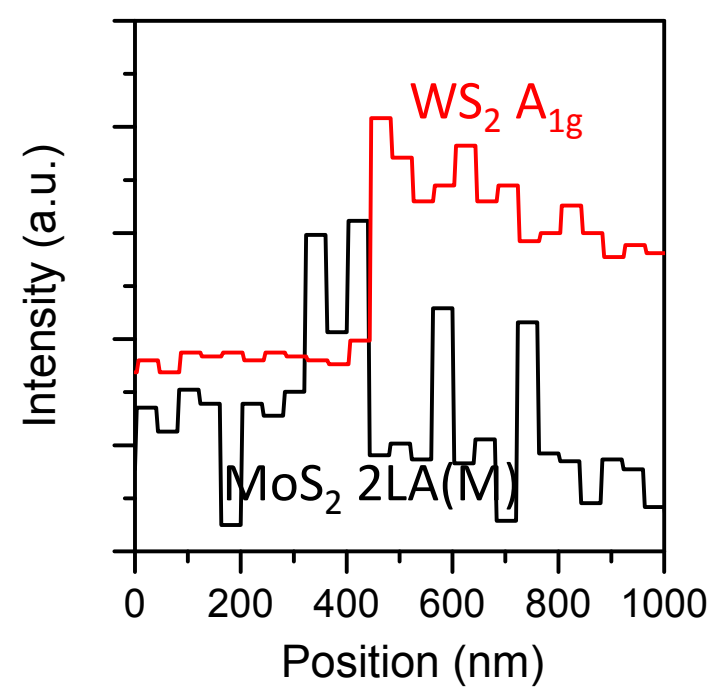

Figure S5 TERS intensity line profile along the marked dashed line in Figure 5(e) using the $\mathrm{WS}_{2}$-like $A_{\lg }$ and $\mathrm{MoS}_{2}$-like $2 L A(M)$ modes. 

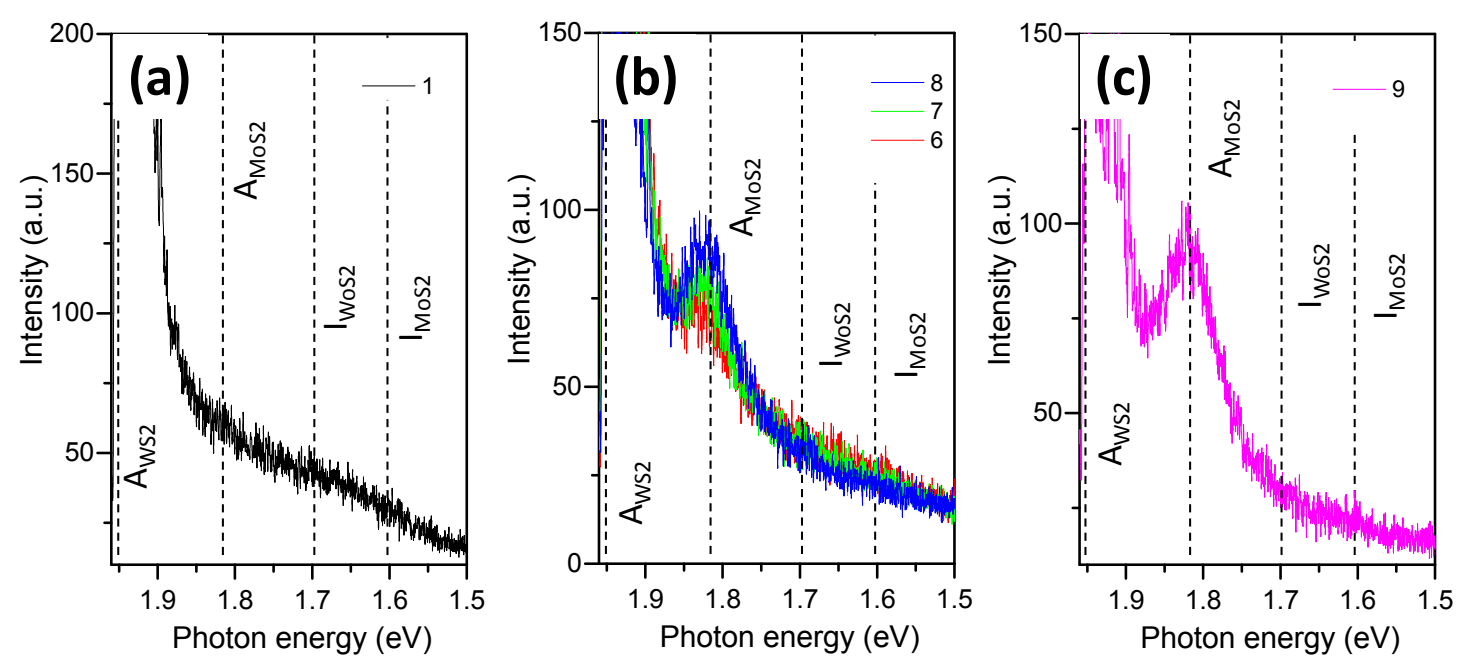

Figure S6 TEPL spectra collected at different positions on HJ II along the dashed lines in Figure 5(h). (a) Point "1" on the $\mathrm{WS}_{2}$ area; (b) points "6"- " 8 " on the $\mathrm{WS}_{2}$ rich alloyed area; (c) point "9" on the $\mathrm{MoS}_{2}$ area. Point "1" shows an indirect transition at $1.7 \mathrm{eV}$. Points " 6 "- " 8 " present the indirect transition of both $\mathrm{WS}_{2}$ at $1.7 \mathrm{eV}$ and $\mathrm{MoS}_{2}$ at $1.6 \mathrm{eV}$. Point "9" presents an indirect transition of $\mathrm{MoS}_{2}$ at $1.6 \mathrm{eV}$. 